\title{
Ein Schatzkästchen tropischer Vielfalt: Die Regenwaldvitrine im Alten Botanischen Garten der Universität Göttingen
}

\author{
Michael Schwerdtfeger
}

\begin{abstract}
An unique showcase with a selection of plants from the lowland tropical rainforests of Central and South America has been restored by dedicated volunteers at the Old Botanical Garden of Göttingen. The showcase includes a selection of poison-arrow frogs, of which the particularly popular strawberry poison-arrow frog (Oophaga pumilio) has now successfully reproduced.
\end{abstract}

\section{Zusammenfassung}

Im Alten Botanischen Garten Göttingen wurde in ehrenamtlicher Arbeit eine Schauvitrine neu gestaltet. Auf nur wenigen Quadratmetern Grundfläche wird nun ein Ausschnitt der reichen Flora aus den Tiefland-Regenwäldern von Mittel- und Südamerika gezeigt. Ergänzend dazu wurden Pfeilgiftfrösche in der Vitrine etabliert. Die bei Terrarianern besonders beliebten Erdbeerfröschchen (Oophaga pumilio) haben sich bereits erfolgreich vermehrt.

\section{Ein historischer Garten mit seinen schönen alten Gewächshäusern}

Der Botanische Garten der Georg-August-Universität Göttingen wurde 1736 angelegt und ist damit so alt wie die Hochschule selbst. Es gibt zwar ältere Botanische Gärten, jedoch nur sehr wenige, die sich seit ihrer Gründung mit gleicher Funktion am gleichen Ort befinden und denen obendrein in den beiden Weltkriegen größere Zerstörungen erspart geblieben sind. So können wir in Göttingen Gewächshäuser besuchen, die vor 160 oder sogar 180 Jahren erbaut wurden. Das Trio unserer drei größten Schauhäuser steht auf Grundmauern, die um 1806 zu Zeiten des sogenannten Königreichs Westfalen unter JÉròme Bonaparte gelegt wurden.

Aber auch die wertvollen Inhalte dieser charmant-historischen Häuser konnten über die Jahrhunderte wachsen: Schon der Gartengründer Albrecht von Haller entdeckte in seiner Studienzeit in Holland sein Interesse an Pflan-

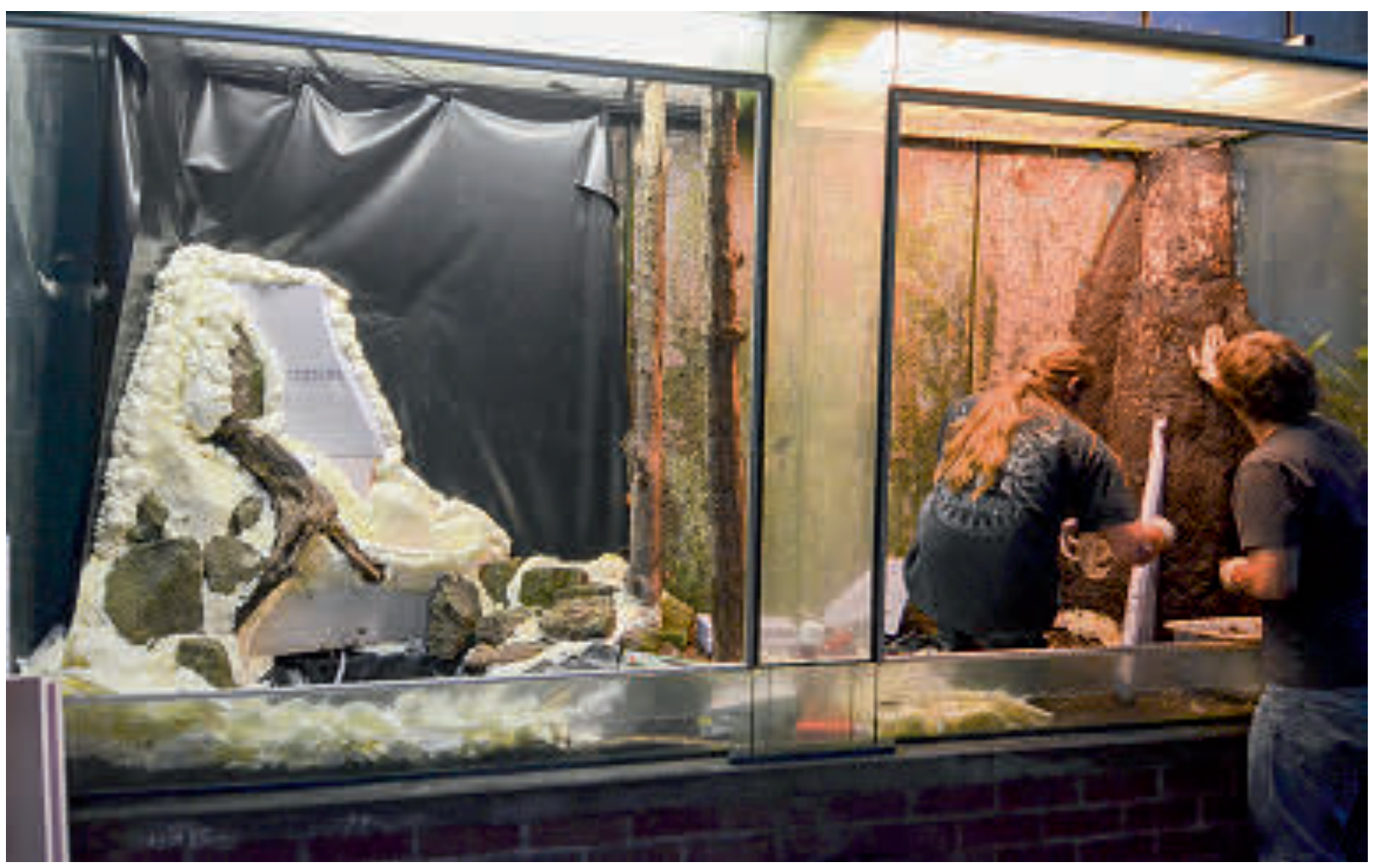


zen aus Übersee, trug im Göttinger Garten „allerhand exotica" zusammen und kultivierte also schon in der Zeit des Rokoko Zitrusgewächse, Palmen und Ananas. Die räumliche Nähe und der kollegiale und freundschaftliche Austausch zwischen den königlichen Gärten in HannoverHerrenhausen und Göttingen begünstigte an beiden Orten den Aufbau wertvollster Sammlungen tropischer Pflanzen. Das 1931 vom Göttinger Gartenmeister Carl Bonstedt herausgegebene Standardwerk „Pareys Blumengärtnerei" zeugt davon, dass der flächenmäßig so kleine Göttinger Garten im Bezug auf den Reichtum seiner Sammlungen bemerkenswert gut aufgestellt war.

\section{Eine neue Schauvitrine}

Im Jahre 2000 hatte die Göttinger Aquarienund Terrarienbaufirma Schwarz dem Botanischen Garten im Regenwaldhaus eine Pflanzenvitrine von $340 \times 170 \times 90 \mathrm{~cm}$ gebaut und durch Einräumen eines großzügigen Freundschaftspreises teilweise gesponsert. Diese Vitrine sollte der zugriff- und diebstahlgeschützten Präsentation von Orchideen und anderen kostbaren Tropengewächsen dienen und war bereits damals so konzipiert, dass sie auch kleine tropische Tiere wie Pfeilgiftfrösche würde beherbergen können. Zunehmende Personalknappheit hat jedoch seither verhindert, dass diese Vitrine ihre eigentliche Bestimmung erfüllen konnte.

Seit Frühjahr 2013 bietet der Botanische Garten für Studierende von Biologie und Biodiversität jährlich eine Costa-Rica-Exkursion an. Bei der ersten dieser Exkursionen nahmen drei Studenten teil, die alle Voraussetzungen aufwiesen, um diesem Projekt nach so langem Dämmerschlaf neues Leben einzuhauchen: Alle drei brachten gute Kenntnis und Begeisterung für tropische Flora und Fauna mit, waren kreative Bastler und außerdem erfolgreiche und erfahrene Terrarianer. Im November 2013 wurde ihnen angeboten, sich bei der Gestaltung dieser Vitrine zu einem riesigen Terrarium zu engagieren. Einzige Vorgabe war, dass die Vitrine geographisch-ökologisch stimmig den Tieflandregenwald Mittel- und Südamerikas zeigen

Abb. 1 (Seite 46): Die neue Vitrine wird gestaltet.

Abb. 2 (unten): Fertig eingerichtete und bepflanzte Vitrine.

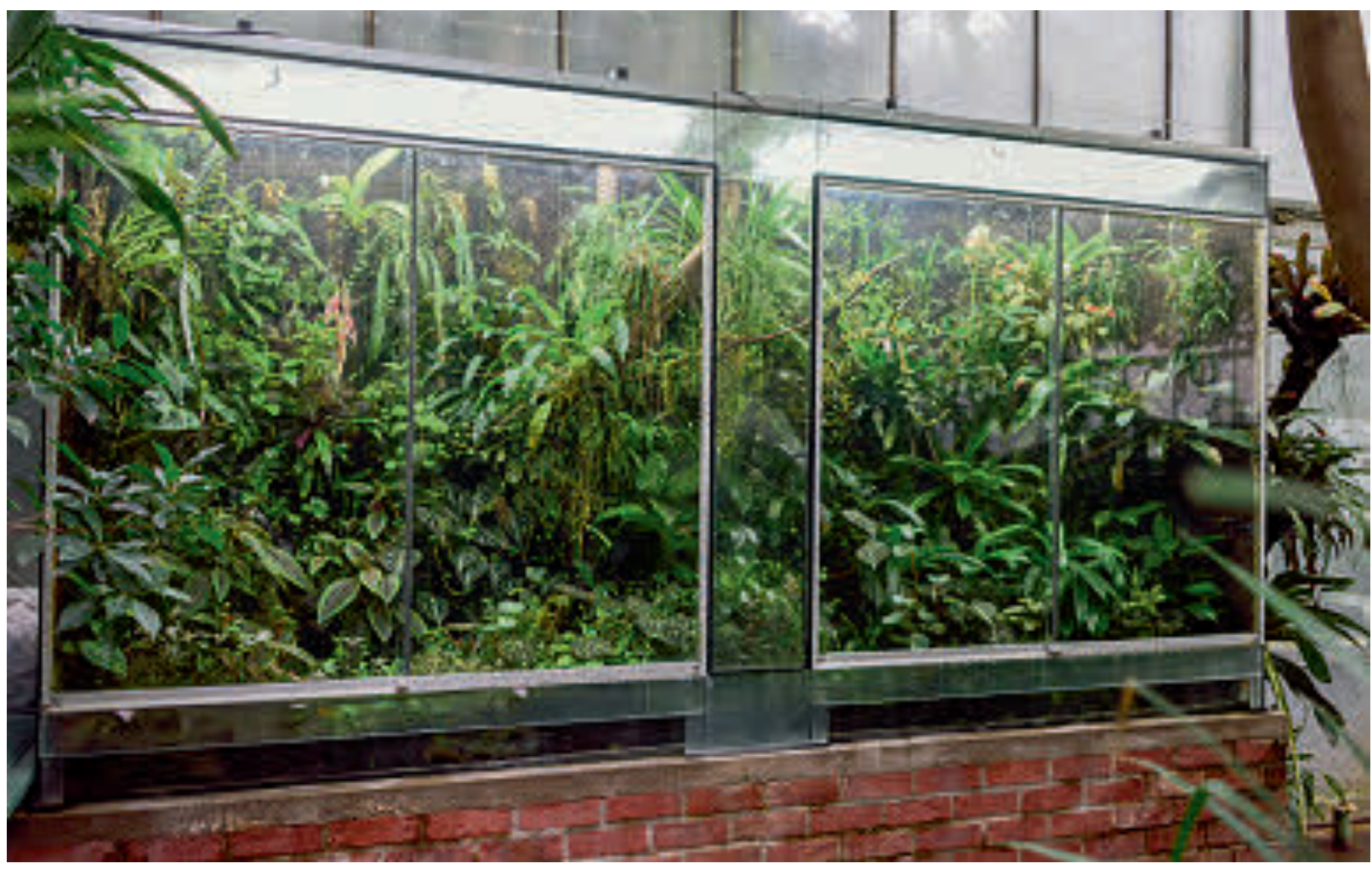



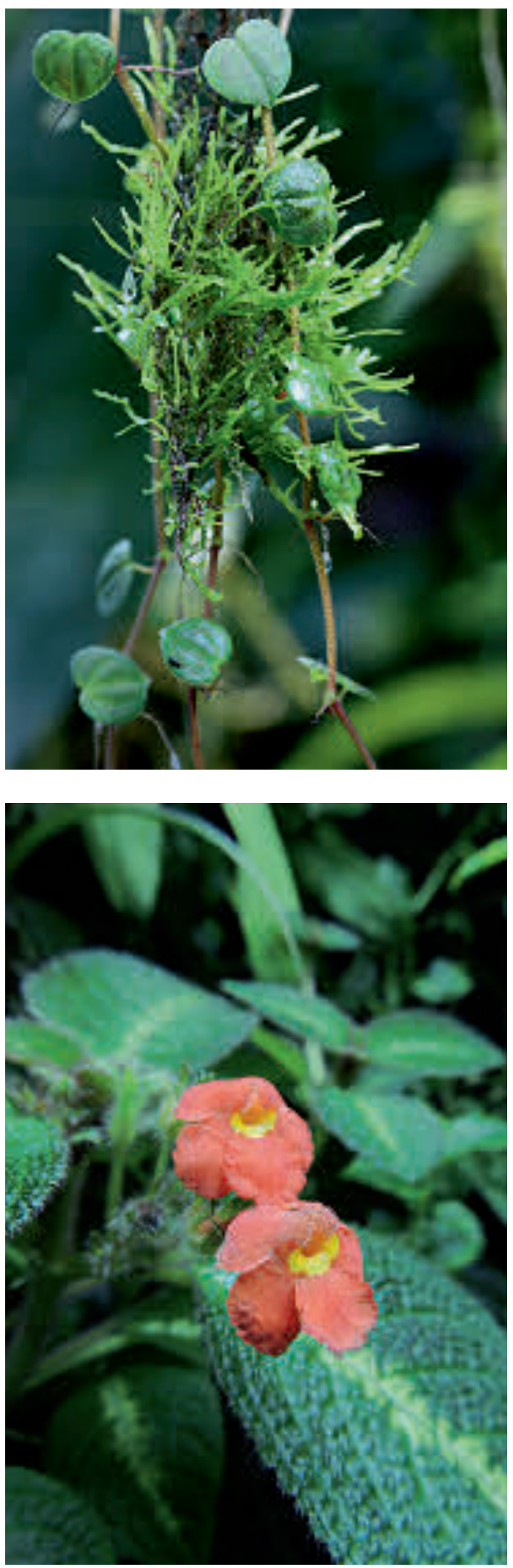

sollte. Alle waren sich einig, bei der Gestaltung und Detailumsetzung den drei jungen Leuten vertrauensvoll freie Hand zu lassen: Das Projekt konnte von den Techniken, Materialien und Ideen der jungen Generation von Terrarianern enorm profitieren.

Die Vitrine wurde vollständig entleert. In wochenlanger, engagierter Feierabend- und Wochenendarbeit entwickelte sich auf einem Grundgerüst aus Steinen, Wurzeln, Bauschaum und Styropor eine Urwaldlandschaft mit Brettwurzeln eines Urwaldriesen, Epiphytenstämmen, einer Felswand und einem kleinen Wasserfall. Dieses zuerst so erschreckend unorganische Skelett bekam eine Übermodellierung aus einem Gemisch aus Torf und Latex-Bindemittel, das nach seinem völligen Abbinden und Durchtrocknen eine poröse, wasseraufnehmende Unterlage für den Pflanzenbewuchs abgeben würde.

Nach einigen Wochen war die Technik komplett: Leuchtstoffröhren sorgten für attraktive und wachstumsfördernde Beleuchtung, eine handelsübliche - allerdings entsprechend groß dimensionierte - Terrarien-Sprühnebelanlage sollte eine angemessene "Regenwaldfeuchte" gewährleisten und nach erfolgter Test- und Einlaufphase die Anlage im Betrieb später fast pflegefrei machen.

\section{Pflanzen für die Vitrine}

Nun konnten im Frühjahr 2014 die Pflanzen Einzug halten, wobei der Alte Botanische Garten Göttingen mit seinen reichen Sammlungen neotropischer Aronstabgewächse, Gesnerien, Farnen, Begonien, Bromelien etc. aus dem Vollen schöpfen konnte. Die Kolleginnen und Kollegen der Königlichen Gärten in HannoverHerrenhausen unterstützten unser Regenwaldvitrine-Projekt mit intensiven Einblicken hinter die Kulissen und in ihre Schatzkammern.

Abb. 3 (oben): Epiphylles Moos auf Peperomia.

Abb. 4 (unten): Episcia cupreata.

Abb. 5 (Seite 49 oben): Dieffenbachia oerstedii.

Abb. 6 (Seite 49 unten): Fittonia verschaffeltii. 
Großzügige Spenden klimatisch geeigneter Orchideen aus ihren artenreichen Sammlungen bereicherten die Bepflanzung der Vitrine.

So konnte die Vitrine bereits im Mai 2014 die unglaubliche Vielfalt neotropischer Tieflandregenwälder abbilden. Über einer Grundfläche von rund $3 \mathrm{~m}^{2}$ wurden ca. 70 Arten an Bodenpflanzen, Lianen und Epiphyten untergebracht, davon viele Kostbarkeiten aus Mexiko, Guatemala, Costa Rica und Ekuador.

Schon kurze Zeit später zeigte sich, dass die Studenten alles richtig gemacht hatten: Dank der guten Planung, der sachkundigen Auswahl, des gärtnerischen „Händchens" bei der Verwendung der Pflanzen und der geschickt eingesetzten Technik waren die Pflanzen wenige Wochen später eingewachsen. Es ließen sich Fotos machen, die genau so auch am natürlichen Standort hätten entstehen können. Das beste Zeugnis einer idealen Klimatisierung war das gute Gedeihen von aus den Tieflandregenwäldern der Karibik und Costa Ricas mitgebrachten epiphyllen (auf lebenden Blättern wachsende) Laubmoosen. Diese entwickelten sich in der Vitrine tatsächlich weiter, sodass hier nun das Wachstum von Epiphyllen auf lebenden Blättern zu beobachten ist.

Von vornherein hatten bei der Bepflanzung der Vitrine Ananasgewächse (Bromelien) eine große Rolle gespielt. Und das nicht nur, weil diese Pflanzenfamilie seit 40 Jahren zu den wertvollsten Spezialsammlungen des Alten Botanischen Gartens gehört, sondern auch ein unverzichtbares Requisit für den authentischen Lebensraum der geplanten tierischen Bewohner (der Pfeilgiftfrösche) ist.

Die ebenmäßig geformten Blattrosetten der Bromelien sind nicht nur von ausgesprochen dekorativem und exotischem Aussehen, sondern sind aufgrund ihrer besonderen Form natürliche Wasserbehälter. Im Zentrum dieser Zisternenbromelien steht auch an den natürlichen Standorten der Pflanzen immer eine kleine Menge Wasser, bei kleinen Formen nur ein Fingerhut voll, bei wagenradgroßen Bromelien oft literweise. Da viele Bromelien als Aufsitzerpflanzen (Epiphyten) auf den Ästen der Bäume wachsen, kann bei einem reich mit Bromelien
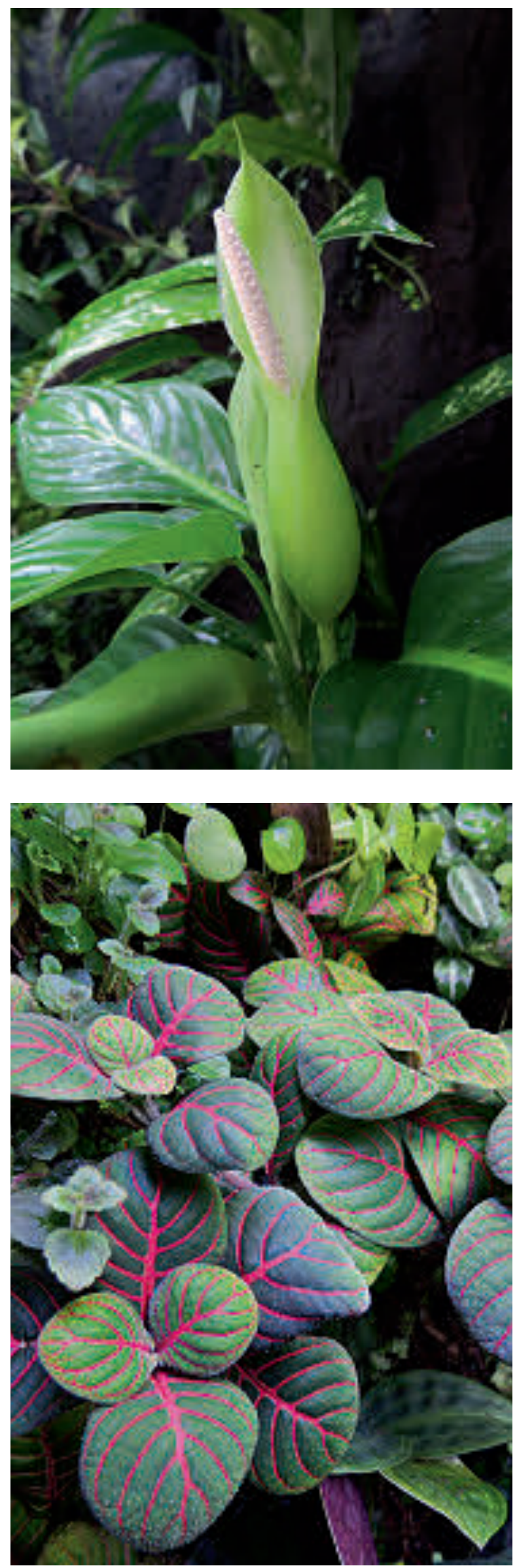


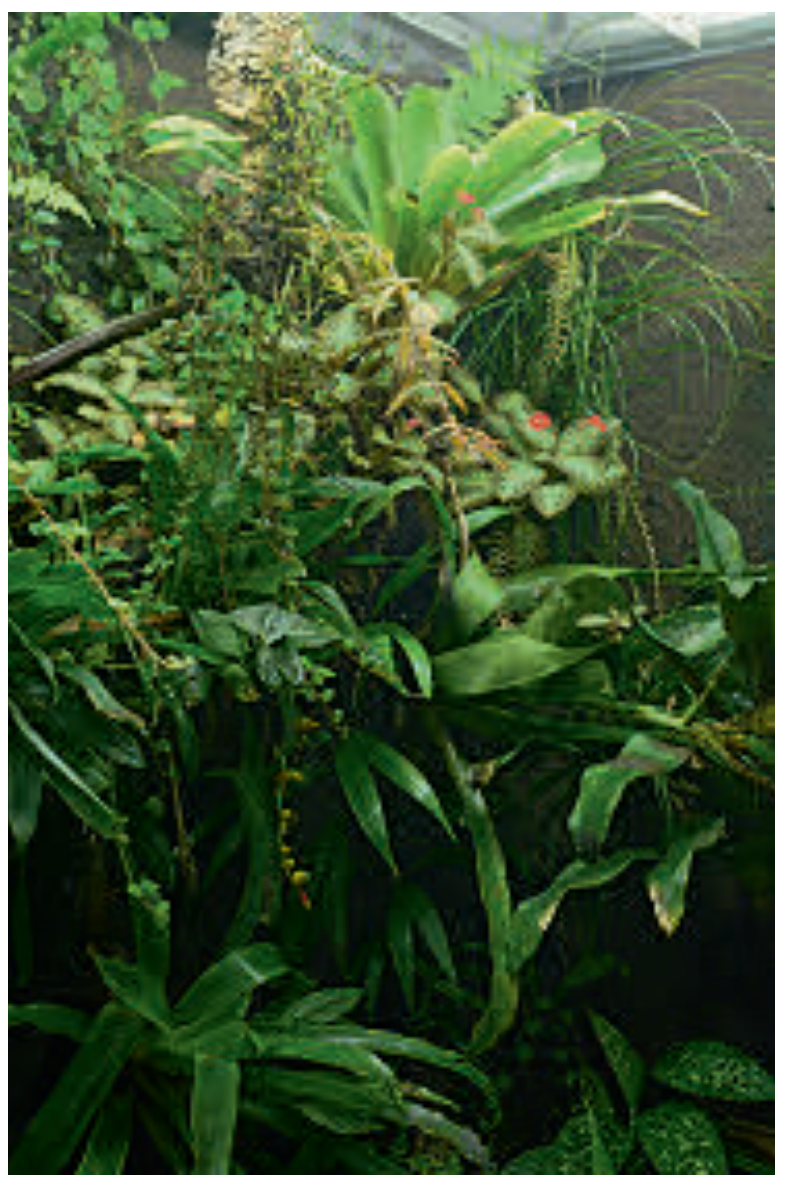

besetzten Urwaldriesen kubikmeterweise Wasser in der Krone verteilt sein, also die Wassermenge ganzer Gartenteiche in der Baumkrone viele Meter über dem Erdboden. An diese hängenden Gärten und schwebenden Teiche hat sich eine reiche Tierwelt angepasst, von baumbewohnenden Mücken, Libellen, Asseln und Regenwürmern über Ameisen, Termiten und Baumschlangen bis zu den faszinierenden knallbunten Dendrobaten, den Pfeilgiftfröschen.

\section{Die Pfeilgiftfrösche ziehen in die Vitrine ein}

Von den ca. 200 in der Natur vorkommenden Arten von Pfeilgiftfröschen sind etliche bei versierten und engagierten Terrarianern in Kultur und werden auch vermehrt. $\mathrm{Zu}$ den begehrtesten und kostbarsten zählt nach wie vor das Erdbeerfröschchen (Oophaga pumilio). Neben den bekannten roten kommen auch blaue, schwarze, gelbe und sonstige Farbformen vor, sodass diese Art in Bezug auf die Farbe als die varia- belste Wirbeltierart der Welt gilt. Allen Erdbeerfröschen gemein ist jedoch die extrem spezialisierte Lebensweise: Die Elterntiere legen ihre wenigen Eier in die wassergefüllten Blatttrichter der Bromelien, holen später die frisch geschlüpften Kaulquappen ab, um sie einzeln huckepack auf dem Rücken in Bromeliengewässer zu verteilen. Sie kehren später alle paar Tage zurück, um jede ihrer Kaulquappen mit unbefruchteten Nähreiern zu versorgen. Erst nach 2-3 Monaten aufopfernder Versorgung durch die Eltern haben die Kaulquappen ihre Entwicklung abgeschlossen und verlassen als winzige Jungfösche die Bromeliengewässer. Der zoologische Name Oophaga („Eierfresser“) bezieht sich darauf, dass sich die Kaulquappen bei dieser Gattung ausschließlich von den Nähreiern ihrer Eltern ernähren und sich, anders als bei vielen anderen tropischen Fröschen, keinesfalls mit Ersatzfutter großziehen lassen. Dieser Umstand macht das Erdbeerfröschchen nach wie vor zu einer kostbaren Art. Nur in einem großen, naturnahen Lebensraum mit reichlich Bromelien können die Männchen ihre Reviere bilden und ihre melodischen, trillernden Lockrufe ertönen lassen, und nur hier können beide Geschlechter ihr einzigartiges Verhaltensrepertoire von Brutfürsorge und Brutpflege ausleben.

Genau diese Voraussetzungen, die der private Terrarianer aus Platzgründen oft nicht bieten kann, waren ja hier gegeben, sodass wir uns im Mai 2014 entschlossen, die Vitrine mit einer Zuchtgruppe von 4 Erdbeerfröschen zu besetzen. Der Erfolg ließ nicht lange auf sich warten: Nach wenigen Tagen ertönten die Werberufe (die für ein nur fingernagelgroßes Tier erstaunlich voluminös sind!), sodass uns seither täglich im Gewächshaus „Regenwaldsound“ erfreut. Bereits im Sommer waren im Becken die ersten Jungtiere zu entdecken, und gesunder Nach-

Abb. 7 (oben): Zwischen Bromelien und anderen Epiphyten fühlen sich Pfeilgiftfrösche wohl.

Abb. 8 (Seite 51 oben): Erdbeerfröschchen in der Tropenvitrine.

Abb. 9 (Seite 51 unten): Die drei Gestalter der Tropenvitrine. Von links nach rechts: LEONARD GEORG, Fabian Mühlberger, Bastian Frank. 
wuchs ist für jeden Tierhalter das beste Zeugnis für optimale Bedingungen und artgerechte Haltung. Immerhin sind sich ernsthafte Aquarianer und Terrarianer bewusst, dass sie mit der Haltung dieser kostbaren Wildtiere (die Haltung von Amphibien muss übrigens behördlich gemeldet sein) eine große Verantwortung übernehmen. Sie sind bestrebt, durch gesunde Nachzucht stabile Populationen in Menschenobhut aufzubauen und illegalen Naturentnahmen damit den wirtschaftlichen Anreiz zu entziehen.

Aber „Terrarianer-Ethik“ ist hier nur ein angenehmer Nebeneffekt. Im Vordergrund steht, dass der Alte Botanische Garten mit seiner neuen Regenwaldvitrine, die durch den ehrenamtlichen Einsatz der drei Studenten zu so einem überaus großen Erfolg wurde, eine weitere Attraktion hat: Einen authentischen Ausschnitt aus dem neotropischen Tieflandregenwald mit seiner ungeheuren Artenfülle und seinem faszinierenden Miteinander von Pflanzen und Tieren.
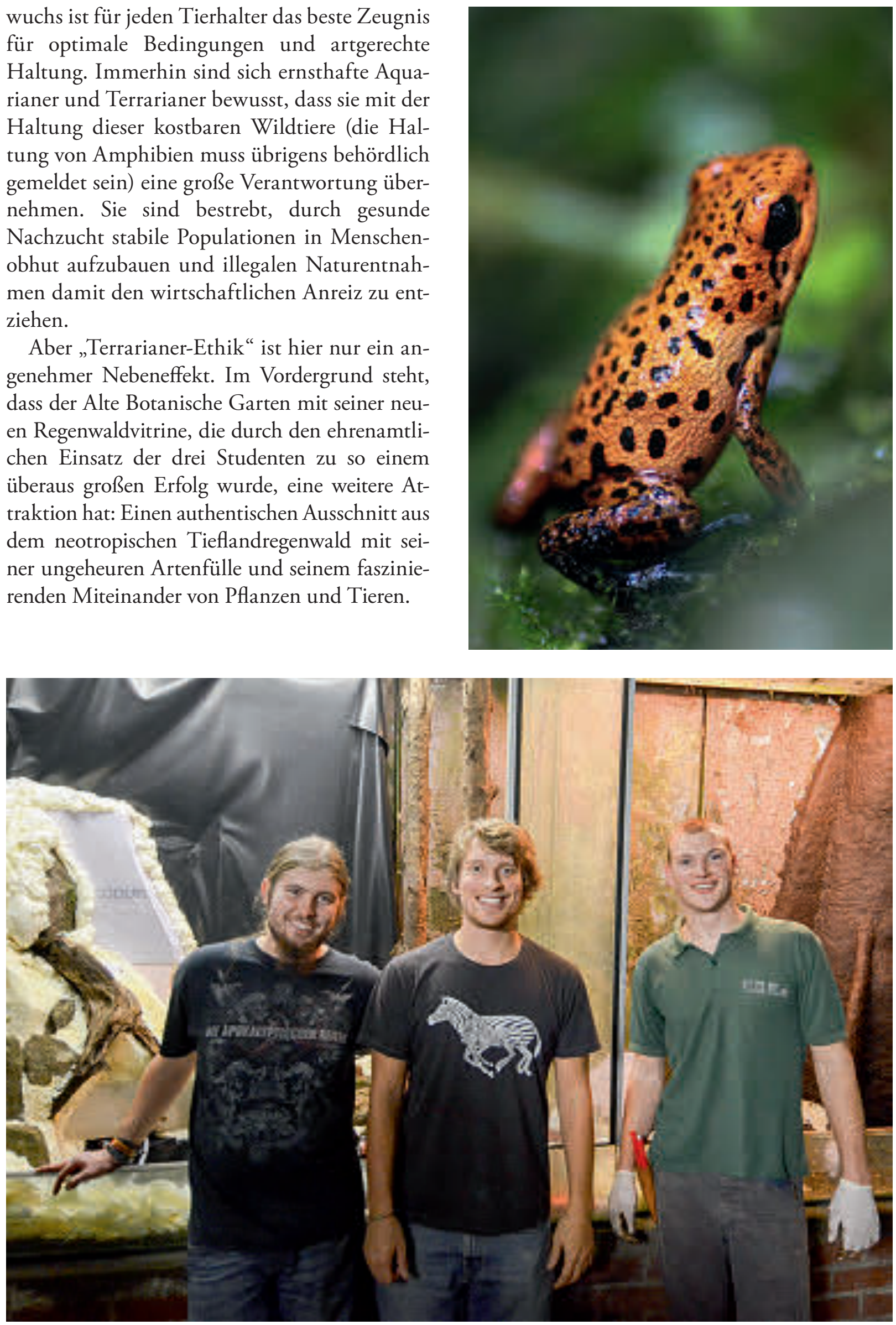